Z Gerontol Geriat 2011 · 44:4-5 DOI 10.1007/s00391-010-0159-9

Online publiziert: 6. Januar 2011

(c) Springer-Verlag 2011

\author{
D. Schaeffer \\ AG Versorgungsforschung/Pflegewissenschaft, Fakultät für \\ Gesundheitswissenschaften, \\ Universität Bielefeld
}

\title{
Patientenaktivierung als Aufgabe der Pflege
}

\section{Interventionsstrategien zur Optimierung der Bewältigung chronischer Krankheit im Alter}

Infolge des voranschreitenden demographisch-epidemiologischen Wandels und der steigenden Zahl an älteren Menschen, die unter chronischen Krankheiten, Multimorbidität und Pflegebedürftigkeit leiden, gewinnen auf Patientenaktivierung setzende Interventionsstrategien, die darauf zielen, die aktive Rolle des Individuums bei der Bewältigung seiner Gesundheits- und Krankheitssituation zu fördern, zunehmend an Bedeutung - auch in der Pflege.

Denn wie in anderen Bereichen der Versorgung macht sich auch dort bemerkbar, wie sehr sich, ausgelöst durch den epidemiologischen Wandel, die Situation und Rolle der Patienten verändert haben. Die Patientenrolle ist bei den heute dominanten chronischen Krankheiten nicht mehr temporär befristet und vorübergehend, wie Parsons dies vor 50 Jahren als zentrale Merkmale beschrieben hat, sondern hat langfristigen Charakter erhalten. Denn anders als Akuterkrankungen, auf die die herkömmliche Patientenrolle zielt, zeichnen sich chronische Erkrankungen ja dadurch aus, dass sie dauerhaft sind und die Erkrankten über lange Zeiträume ihres Lebens hinweg begleiten. Das gilt umso mehr, als sich infolge des medizinischpharmakologischen Fortschritts die Verlaufsdauern bei chronischer Krankheit enorm ausgedehnt haben. Für die Erkrankten bedeutet dies, dass sie länger als früher mit chronischer Krankheit (über-) leben. Aber dem Gewinn an Jahren steht gegenüber, dass sie auch lange Zeit mit Krankheit leben müssen und gezwungen sind, sie in ihr Leben zu integrieren und ihr Leben auf die Krankheit abzustellen.

Ähnliche Herausforderungen zeigen sich auch auf anderer Ebene: So sind die Behandlungs-, aber auch Informationsund Partizipationsmöglichkeiten, etwa bei Therapie- und Versorgungsentscheidungen, in den vergangenen Jahren deutlich gestiegen. Auch diese Entwicklung hat die herkömmliche Patientenrolle verändert: Sie ist weniger passiv, sondern weist mehr aktive Elemente und neue Gestaltungsspielräume auf.

$\mathrm{Zu}$ den Besonderheiten chronischer Erkrankung gehört außerdem, dass die mit ihnen verbundenen Bewältigungsherausforderungen nicht gleichförmig sind, sondern sich von Phase zu Phase im Krankheitsverlauf anders ausnehmen. Auch dies ist nicht ohne Auswirkungen auf die Patientenrolle geblieben. Diese ist damit einhergehend vielgestaltiger und anspruchsvoller geworden und fordert den Erkrankten heute ein hohes Maß an Flexibilität, Eigenkompetenz und Selbstmanagementvermögen ab. Fragt sich, ob alle Patienten dem zu entsprechen vermögen, und falls nein, wie sie in geeigneter Weise gefördert werden können, sodass sie diesem Anforderungswandel gerecht werden. 
Patientenaktivierung setzt hier an und kann im Sinn des Empowerments auf die Stärkung der Handlungsfähigkeit und Selbstwirksamkeit zielen oder sich der Vermittlung erforderlichen Krankheitsund Gesundheitswissens („health literacy") widmen, das chronisch Kranke benötigen, um ihre Situation konstruktiv und eigenaktiv zu bewältigen. Patientenaktivierung kann aber ebenso auf Kompetenz- und Ressourcenförderung durch Training und Coaching zielen oder als settingorientierte Intervention angelegt sein und sich mit der Gestaltung der Umgebung befassen.

In jedem Fall sind patientenaktivierende Konzepte durch strikte Patientenorientierung gekennzeichnet, folgen also in erster Linie dem Bedarf und der Problemsicht der Patienten bzw. derjenigen, die unter Krankheit, Funktionseinschränkungen und Pflegebedürftigkeit leiden, und sind nicht etwa an systemischen Zwängen oder der Expertenperspektive orientiert. Wie breit das Spektrum an Themen und Interventionsstrategien ist, deuten die nachfolgenden Beiträge an, die auf Projekte des Pflegeforschungsverbunds NRW zurückgehen ${ }^{1}$. Es reicht von Selbstmanagementunterstützung über Empowerment und Kompetenzförderung durch Storytelling und Erfahrungsaustausch, Biographiearbeit zur Stimulierung positiver Emotionen und Verbesserung des emotionalen Wohlbefindens - eine gerade bei kognitiv eingeschränkten Menschen wichtige Aufgabe - bis hin zur Vermeidung von Selbst- und Fremdgefährdung durch die Herstellung einer ressourcenerhaltenden Umgebung.

Samt und sonders zeigen die Beiträge, wie wichtig es ist, auch hierzulande das Interventionsspektrum der Gesundheits-

\footnotetext{
1 Der Pflegeforschungsverbund NRW gehört zu den drei vom Bundesministerium für Bildung und Forschung (BMBF-Förderkennziffer Phase Il: 01GT0615) von 2004 bis 2010 bundesweit geförderten Pflegeforschungsverbünden. Ziel des NRW-Forschungsverbunds ist es, patientenorientierte Pflegekonzepte zur Verbesserung der Bewältigung chronischer Krankheit zu erarbeiten, erproben und evaluieren. Mein Dank gilt hier Dr. Annett Horn, der die Geschäftsführung obliegt und die auch die Koordination der Beiträge übernommen hat.
}

professionen und so auch der Pflege um Aufgaben der Patientenaktivierung zu erweitern.

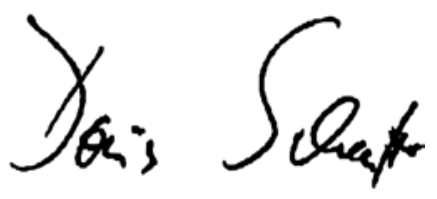

Doris Schaeffer

\section{Korrespondenzadresse}

Prof. Dr. D. Schaeffer

AG Versorgungsforschung/Pflegewissenschaft Fakultät für Gesundheitswissenschaften Universität Bielefeld

Postfach 100131

33501 Bielefeld

doris.schaeffer@uni-bielefeld.de 